\title{
Study of Suspension Bridge Deformation Using Neural Network
}

\author{
Dr. Boris Mazurov \\ Department of physical geodesy and remote sensing \\ SSUGT \\ Russian Federation \\ btmazurov@mail.ru
}

\author{
Dr. Fawzi Zarzoura \\ Faculty of Engineering \\ Mansoura University \\ Egypt
}

\author{
Dr. Mohamed Hassan \\ Faculty of Engineering \\ Mansoura University \\ Egypt
}

\begin{abstract}
Suspension bridges are an important and widely used element of regional and urban infrastructure for traffic and transportation. One of the elements of the system to ensure their safety and security is the geodetic monitoring by using GNSS technologies. Displacement of the bridges points depend not only on time, the impact of traffic capacity and wind on the naturaltechnical system of suspension bridge should be taken into consideration. This makes the task of constructing a predictive model difficult. The most significant effects are the temperature change, the impact of wind and vehicle movement. Here is some analysis of these effects on the dynamics of a suspension bridge using real experimental data. Two analytical methods, namely Neural Networks (NN) and Least Square (LS), were used for the prediction of the effects on the behavior of suspension bridges. The results of the Neural Networks give better picture than the results of Least Square.
\end{abstract}

Keywords- suspension bridges, GNSS, deformation, neutral networks, least square

\section{INTRODUCTION}

Bridges are important transport nodes and play a vital role in socio-economic development. Suspension bridges are a type of bridges in which the deck is hung below suspension cables on vertical suspenders. Cables suspended between towers, plus vertical suspender cables that carry the weight of the deck below, upon which traffic crosses. The suspension cables must be anchored at each end of the bridge, since any load applied to the bridge is transformed into a tension in these main cables. The main cables continue beyond the pillars to decklevel supports, and further continue to connections with anchors in the ground. The roadway is supported by vertical suspender cables or rods, called hangers. The bridge will usually have two smaller spans, running between either pair of pillars, and the highway, which may be supported by suspender cables or may use a truss bridge to make this connection. Bridge safety will become an even more serious problem faced by bridge authorities.
The Global Navigation Satellite System (GNSS) has been utilized for more than two decades in the deformation monitoring of a variety of structures, such as dams, building, bridges, slopes, etc., around the world [6]. With the technological advent of GPS positioning, telecommunications, and signal processing as well as public awareness, GPS has been widely tested in recent years for monitoring slender structures such as large suspension bridges and high-rise buildings and gradually becomes an alternative tool for structural health monitoring (SHM) [6]. GPS-based bridge monitoring has many appealing advantages over more traditional bridge monitoring sensor systems. For instance, GPS monitoring could be carried out in a real-time and in automatic manner for the provision of timely geometric displacements under different weather conditions [9].

The integration of GPS and inertial sensors is typically accomplished through using Kalman Filter (KF) [5]. KF, introduced in 1960 by Dr. R. E. Kalman [3], is a statistical technique that combines the statistical nature of system errors with system dynamics, as represented by a state space model, to achieve best estimation of the system state. The KF is considered as an optimal filter [5]. The KF and Least Square (LS) are two types of wavelet analysis used in a wide area of GPS applications. It was found that the calculated coordinates from LS and KF analysis methods are much closer [7]. However, the focus of this research is to show the differences between the use of NN (Neural Network), LS and Chebyshev polynomials in GPS data processing. 


\section{BRIDGE DESCRIPTION}

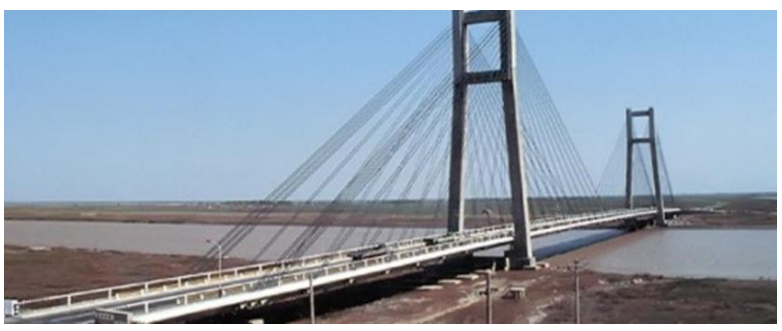

Fig. 1. Tianjin Yonghe Bridge

Tianjin Yonghe Bridge is a road bridge, which crosses the new Yongding River. Located in the eastern suburb of Tianjin in China, it is the vital channel of Shanguang highway. Its total length is $512.4 \mathrm{~m}$, adopting the prestressed concrete cable-stayed bridge with double pylons, which main span is $260 \mathrm{~m}$. The entire width of the bridge deck is $13.6 \mathrm{~m}$, including lane road of $9 \mathrm{~m}$ and the pavement on both sides of the bridge. The bridge is a five-span continuous prestressed concrete cable-stayed bridge with the main span of $260 \mathrm{~m}$, double pylons and double columns [7].

The execution date was from 30 October 1987 to 27 January 1990; and it was closed in October 2006, because of cracks emerged over the mid span, and opened in August 2007, after its rehabilitation [8]. The GPS observations are Real Time Kinematic (RTK) with the differential GPS (DGPS) system. The receivers were considered along the bridge, every rover station was observed for $24 \mathrm{hr}$. The number of data collection is $72000 / \mathrm{hr}$, and each epoch is corrected with the base station [1].

\section{MATHEMATICAL MODEL}
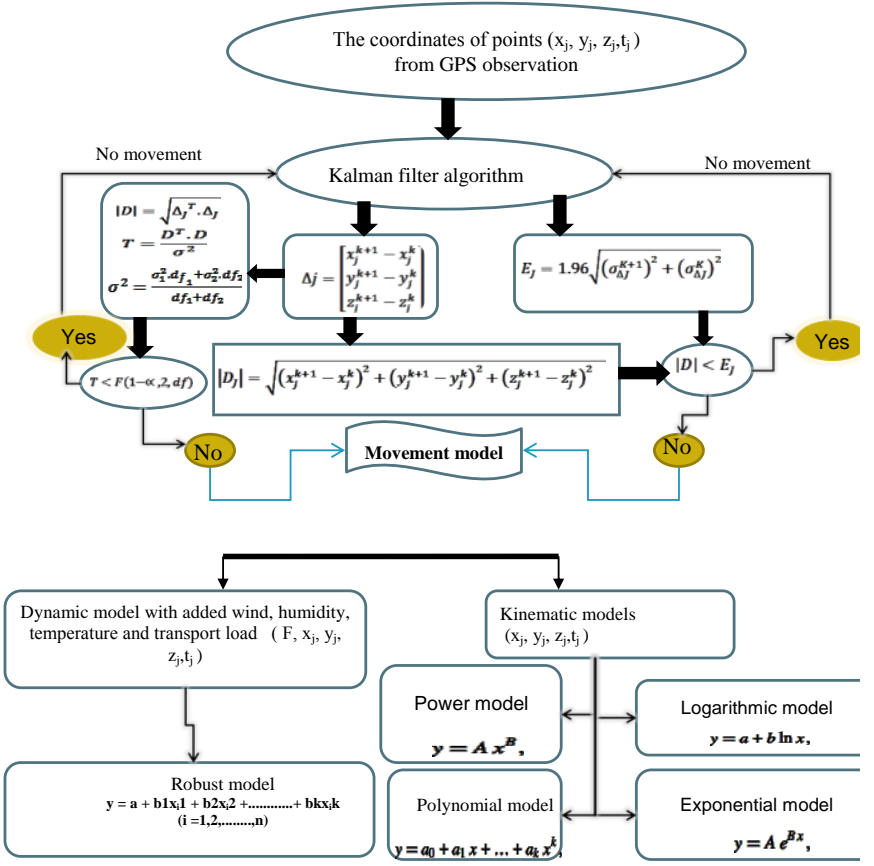

IV. LEAST SQUARE TECHNIQUE

$$
\begin{aligned}
& \frac{d \Delta}{d a_{n}}=\left(t_{i}-t_{0}\right)^{n} \quad, \frac{d \Delta}{d a_{o}}=1 \\
& A \cdot \Delta+L=V \\
& A=\left[\begin{array}{cccc}
\left(t_{1}-t_{0}\right)^{n} & \left(t_{1}-t_{0}\right)^{n-1} & \cdots & 1 \\
\vdots & \vdots & \ddots & \vdots \\
\vdots & \vdots & \ddots & 1 \\
\left(t_{i}-t_{0}\right)^{n} & \left(t_{i}-t_{0}\right)^{n-1} & \cdots & 1
\end{array}\right], L=\left[\begin{array}{l}
\Delta_{1} \\
\Delta_{2} \\
\vdots \\
\Delta_{n}
\end{array}\right]
\end{aligned}
$$$$
\left[\begin{array}{l}
a_{0} \\
a_{1} \\
\vdots \\
a_{n}
\end{array}\right]=\left(A^{T} \cdot A\right)^{-1} \cdot\left(A^{T} \cdot L\right)
$$

\section{CHEBYSHEV POLYNOMIALS}

Chebyshev polynomials $T_{n}$ or $U_{n}$ are polynomials of degree $\mathrm{n}$ and the sequence of Chebyshev polynomials of either kind composes a polynomial sequence. Chebyshev polynomials are polynomials with the largest possible leading coefficient. They are also the external polynomials for many other properties.

Chebyshev polynomials are important in the approximation theory because the roots of the Chebyshev polynomials of the first kind, which are also called Chebyshev nodes, are used as nodes in polynomial interpolation. The resulting interpolation provides an approximation that is close to the polynomial of the best approximation to a continuous function under the maximum norm.

An arbitrary polynomial of degree $N$ can be written in terms of the Chebyshev polynomials of the first kind. Such polynomial $p(x)$ is of the form:

$$
p(x)=\sum_{n=0}^{N} a_{n} T_{n}(x) .
$$

\section{NEURAL NETWORKS TECHNIQUE}

An Artificial Neural Network (ANN) is an information processing paradigm that is inspired by the way biological nervous systems, such as the brain, process information. The key element of this paradigm is the novel structure of the information processing system. It is composed of a large number of highly interconnected processing elements (neurons), working in unison to solve specific problems. ANNs, like people, learn by example. An ANN is configured for a specific application, such as pattern recognition or data classification, through a learning process. Learning in biological systems involves adjustments to the synaptic connections that exist between the neurons [2]

An artificial neuron is a device with many inputs and one output. The neuron has two modes of operation: the training mode and the using mode. In the training mode, the neuron can be trained to fire (or not), for particular input patterns. In the using mode, when a taught input pattern is detected at the input, its associated output becomes the current output. If the input pattern does not belong in the taught list of input 
patterns, the firing rule is used to determine whether to fire or not [3].

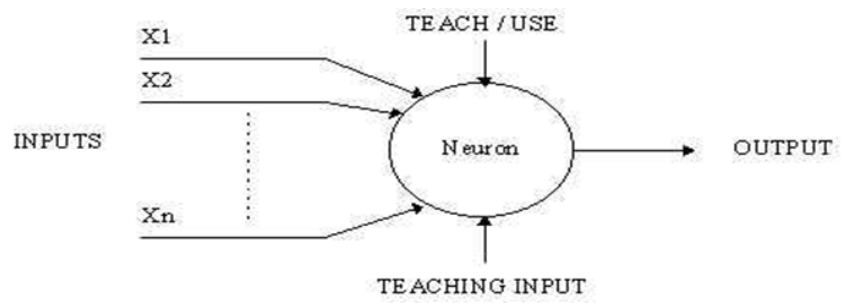

Fig. 2. A simple neuron

The behavior of an ANN depends on both the weights and the input-output function (transfer function) that is specified for the units. This function typically falls into one of three categories:

Linear output is proportional to the total weighted output.

Threshold of output is set at one of two levels, depending on whether the total input is greater or less than some threshold value.

Sigmoid output varies continuously but not linearly as the input changes. Sigmoid units bear a greater resemblance to real neurons than linear or threshold units do, but all three must be considered as rough approximations.

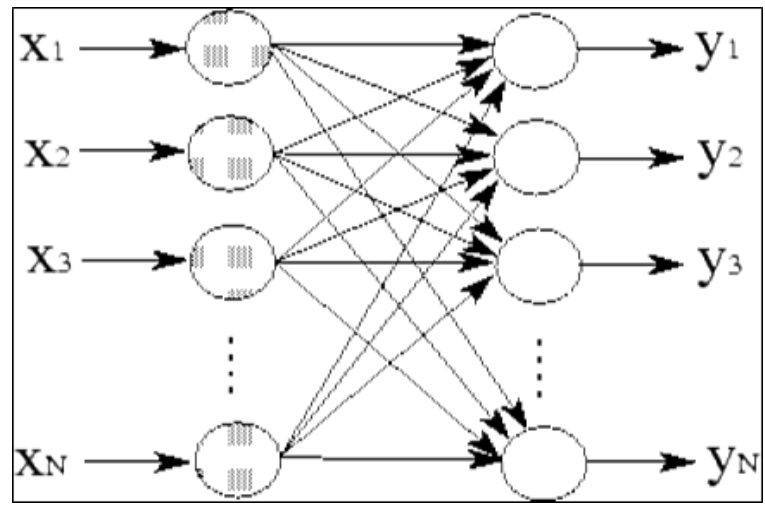

Fig. 3. Single-Layer Network

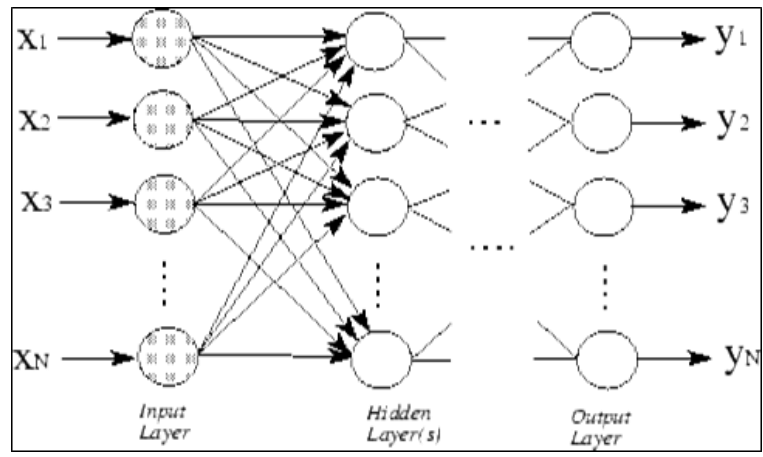

Fig. 4. Multilayer Network

The authors' basic computational element (model neuron) is often called a node or unit. It receives the input from some other units, or perhaps from an external source. Each input has associated weight $w$, which can be modified so as to model synaptic learning. The unit computes some function $f$ of the weighted sum of its inputs:

$$
y_{i}=f\left(\sum_{j} w_{i j} y_{j}\right)
$$

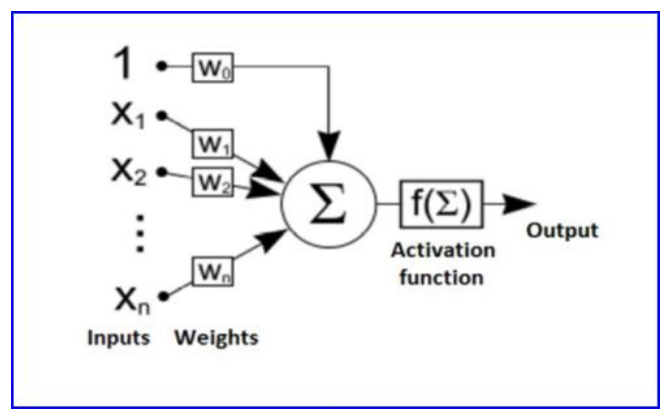

Fig. 5. Neural network system

Its output, in turn, can serve as an input to other units.

- The weighted sum is called the net input to unit $i$, often written as net $i$.

- $\quad$ Let us note that $w_{i j}$ refers to the weight from unit $j$ to unit $i$ (not the other way around).

- Function $f$ is the unit's activation function. In the simplest case, $f$ is the identity function, and the unit's output is just its net input. This is called a linear unit.

The following algorithm is proposed:

1. Initialize all weights to small random values.

2. REPEAT until done.

1. For each weight $w_{i j}$

2. For each data point $(x, t) \mathrm{p}$.

1. $\quad$ set input units to $x$.

2. compute the value of output units.

3. for each weight $w_{i j}$.

3. For each weight $w_{i j}$.

The algorithm terminates once one is at, or sufficiently near to, the minimum of the error function, where $G$ (gradient) $=0$. Then it is possible to say that the algorithm has converged [3].

\section{OBSERVATIONS AND ANALYSIS}

This section discusses the analysis process of the GPS observations and the bridge towers movement. In addition, the statistical analyses will discuss:

\begin{tabular}{|c|c|c|c|c|c|c|c|}
\multicolumn{7}{c|}{ TABLE I. THE MEAN OF OBSERVATIONS } \\
\begin{tabular}{|c|c|c|c|c|c|}
\hline \\
DATE
\end{tabular} & $\begin{array}{c}6- \\
2007\end{array}$ & $\begin{array}{c}8- \\
2007\end{array}$ & $\begin{array}{c}10- \\
2007\end{array}$ & $\begin{array}{c}1- \\
2008\end{array}$ & $\begin{array}{c}2- \\
2008\end{array}$ & $\begin{array}{c}2008 \\
(\mathrm{CHEC} \\
\mathrm{K})\end{array}$ & $\begin{array}{c}2008 \\
(\mathrm{CHEC} \\
\mathrm{K})\end{array}$ \\
\hline $\mathrm{D} X_{\text {1MEAN }}(\mathrm{cm})$ & 0.3 & -0.2 & 2.4 & 8.3 & 8.8 & 5.4 & -0.9 \\
\hline $\mathrm{D} Y_{1 \text { MEAN }}(\mathrm{cm})$ & -0.1 & 0.5 & 1.5 & 6.0 & 5.9 & 4.8 & -0.1 \\
\hline $\mathrm{D} X_{\text {2MEAN }}(\mathrm{cm})$ & 0.4 & 1.7 & -0.7 & -0.9 & -0.7 & -0.2 & 1.8 \\
\hline $\mathrm{D} Y_{\text {2MEAN }}(\mathrm{cm})$ & 0.5 & -1.3 & -0.9 & -1.0 & -1.4 & -2.9 & 1.2 \\
\hline
\end{tabular}


The $R^{2}$ value measures how much of the variation in the data points can be explained by the selected regression model:

$$
R^{2}=\frac{S S R}{S S T}=1.0-\frac{S S E}{S S T} \quad 0 \leq R^{2} \leq 1,
$$

where

$$
\begin{array}{ll}
S S R=\sum_{i=1}^{n}\left(\hat{Y}_{i}-\bar{Y}\right)^{2} & \text { (The regression sum of squares) } \\
S S E=\sum_{i=1}^{n}\left(Y_{i}-\hat{Y}\right)^{2} & \text { (The residual or error sum of }
\end{array}
$$
squares)

$$
S S T=\sum_{i=1}^{n}\left(Y_{i}-\bar{Y}\right)^{2}
$$

(The total sum of squares, SST=

$\mathrm{SSE}+\mathrm{SSR}$

and $\hat{Y}_{i}$ represents the $i^{\text {th }}$ fitted value (calculated using the selected model) of dependent variable $Y$.

Table (2) shows the computed equation from results and value of every equation.

TABLE II. THE COMPUTED EQUATION FROM RESULTS AND VALUE OF EVERY EQUATION

\begin{tabular}{|c|c|c|}
\hline $\begin{array}{c}\text { TYPE OF } \\
\text { EQUATION }\end{array}$ & EQUATION & $R^{2}$ \\
\hline Linear & $x=0.012 t-0.012$ & 0.909 \\
\hline Poly 2nd & $x=0.001 t^{2}-7 * 10^{-5} t+0.000$ & 0.970 \\
\hline Poly 3rd & $x=0.00 t^{3}+0.008 t^{2}-0.017 t+0.003$ & 0.998 \\
\hline Poly 4th & $x=-5 * 10^{-5} t^{4}-0.0001 t^{3}+0.003 t^{2}-0.011 \mathrm{t}+0.002$ & 1.000 \\
\hline Linear & $y=0.008 t+0.008$ & 0.931 \\
\hline Poly 2nd & $y=0.000 t^{2}+0.002 t-0.002$ & 0.965 \\
\hline Poly 3rd & $y=0.000 t^{3}+0.003 t^{2}-0.006 t+0.000$ & 0.979 \\
\hline Poly 4th & $\mathrm{y}=-0.0001 t^{4}+0.002 t^{3}-0.008 t^{2}+0.012 t-0.001$ & 1.000 \\
\hline Linear & $x=-0.002 t+0.009$ & 0.507 \\
\hline Poly 2nd & $x=0.000 t^{2}-0.001 t+0.008$ & 0.512 \\
\hline Poly 3rd & $x=0.000 t^{3}-0.005 t^{2}+0.013 t+0.005$ & 0.836 \\
\hline Poly 4th & $x=0.0001 t^{4}-0.003 t^{3}+0.018 t^{2}+0.032 t+0.003$ & 1.000 \\
\hline Linear & $y=-0.001 t+0.000$ & 0.583 \\
\hline Poly 2nd & $y=0.000 t^{2}-0.005 t+0.002$ & 0.749 \\
\hline Poly 3rd & $y=0.000 t^{3}+0.003 t^{2}-0.013 t+0.004$ & 0.983 \\
\hline Poly 4th & $y=3 * 10^{-5} t^{4}-0.0001 t^{3}+0.006 t^{2}-0.017 t+0.004$ & 1.000 \\
\hline
\end{tabular}

From table (II) for the towers, the authors suggest using polynomial 4th when predicting the deformation with time because the value of $R^{2}=1.00$

When the authors used it in prediction, the next two values differences in the observation and prediction values were very high. It means that the prediction values did not depend only on time. It is necessary to take traffic loads and wind into consideration.

From Chebyshev polynomials, these equations were obtained for prediction of:

$$
\begin{aligned}
& f(t)=a_{0}+a_{1} t+a_{2} t^{2}+a_{3} t^{3}+a_{4} t^{4} \\
& \text { for } x l \rightarrow f(t)=3.388+0.974 t+0.143 t^{2}+0.017 t^{3}+0.003 t^{4} \\
& \text { for } y l \rightarrow f(t)=2.389+0.685 t+0.097 t^{2}+0.012 t^{3}+0.002 t^{4} \\
& \text { for } x 2 \rightarrow f(t)=0.007-0.084 t-0.013 t^{2}+0.002 t^{3}+0.001 t^{4}
\end{aligned}
$$

$$
\text { for } y 2 \rightarrow f(t)=-0.730-0.173 t-0.022 t^{2}-0.003 t^{3}-0.001 t^{4}
$$

TABLE III. THE DIFFERENCE IN VALUES FROM OBSERVATIONS AND

\begin{tabular}{|c|c|c|c|c|}
\hline DATE & $\begin{array}{c}4-2008 \\
\text { EAST SQUARR }\end{array}$ & $\begin{array}{c}6-2008 \\
\text { LEAST } \\
\text { SQUARE }\end{array}$ & $\begin{array}{c}4-2008 \\
\text { CHEBYSHEV } \\
\text { POLYNOMIALS }\end{array}$ & $\begin{array}{c}6-2008 \\
\text { CHEBYSHEV } \\
\text { POLYNOMIALS }\end{array}$ \\
\hline $\mathrm{D} X_{1}(\mathrm{~m})$ & -0.109 & -0.562 & 0.773 & 1.332 \\
\hline $\mathrm{D} Y_{2}(\mathrm{~m})$ & 0.319 & 0.373 & 0.534 & 0.92 \\
\hline $\mathrm{D} X_{2}(\mathrm{~m})$ & 0.123 & -0.131 & -0.068 & -0.12 \\
\hline $\mathrm{D} Y_{2}(\mathrm{~m})$ & 0.634 & 1.113 & -0.122 & -0.206 \\
\hline
\end{tabular}

A continuous monitoring system was designed by using GPS observation and Neural Networks technique under certain external forces. A multilayer feed-forward neural network, using Levenberg-Marquardt learning algorithm, was applied. The steps of the entire study can be summarized as follows:

- Data collection and preprocessing

- Generation of the training patterns

- Identification of the network architecture

- Optimization of the network parameters and validation of the network by using a test data set.

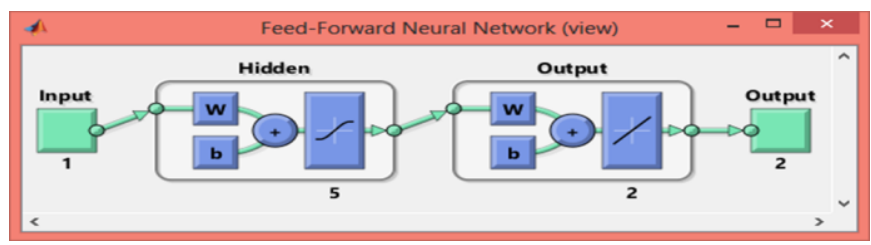

Fig. 6. The neural network model

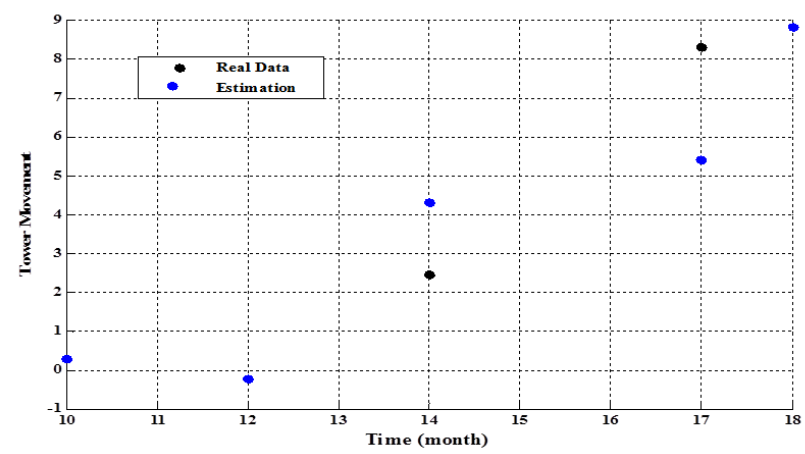

Fig. 7. The differences between the real and estimation values for the first tower 


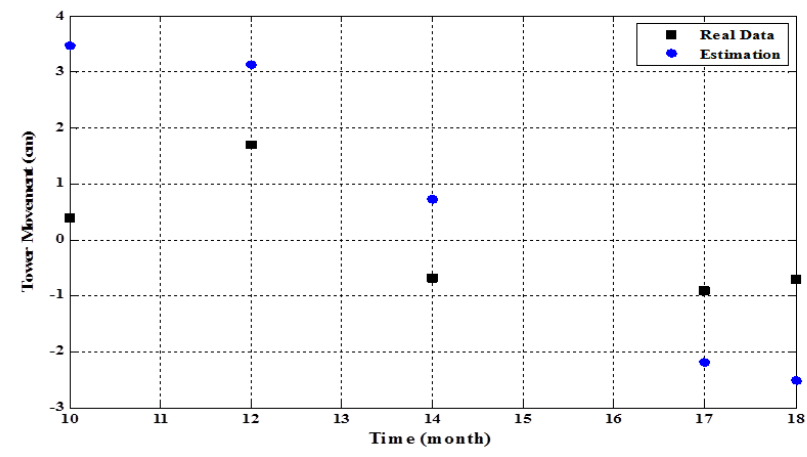

Fig. 8. The differences between the real and estimation values for the second tower

The results given in figures 7 and 8 show that a very good approximation can be obtained even if the input data sampling rate is very low. The more frequently the input data is measured, the better approximation can be obtained by using neural network methods. The maximum differences between the real and estimated values equal $3 \mathrm{~cm}$.

\section{CONCLUSIONS}

General information about the movements of a suspension bridge can be obtained using frame work of mathematical models that will provide information about action and reaction quantities over time. The proposed surveying techniques, using DGPS and RTK, can provide valuable deformation data of the structural members. Using of Neural Network technique in prediction the movements of bridges better than Chebyshev polynomials and the Least Square technique.
The prediction values did not depend only on time. It is necessary to take traffic loads and wind into consideration. Neural networks can be considered as a technique for the description of movements, especially in continuous monitoring of suspension bridges.

\section{References}

[1] R.S. Azar, H.Z. Shafri, "Mass structure deformation monitoring using low cost differential global positioning system device", J. Applied Sciences, ASCE, Vol. 6, No.1, pp. 152-156, 2009.

[2] A. Eichhorn, "Tasks and Newest Trends in Geodetic Deformation Analysis: A Tutorial", 15th European Signal Processing Conference (EUSIPCO 2007), Poznan, Poland, September 3-7, pp. 1156-1160, 2007.

[3] S. Haykin, Kalman filtering and neural networks, Communication Research Laboratory, McMaster University, Hamilton, Ontario, Canada, 2001, 284 p., ISBNs: 0-471-36998-5 (Hardback); 0-47122154-6 (Electronic).

[4] H. Martin, Matlab Recipes for Earth Sciences, second ed., Springer, Berlin Heidelberg, New York, 279 p., 2007.

[5] D. Kaplan, J. Hegarty, Understanding GPS Principles and Applications, Second Edition, Artech House, Inc., UK, 685 Canton Street, Norwood, MA 02062, 706 p., 2006, International Standard Book Number: 1-58053-894-0.

[6] X. Meng, Real-time deformation monitoring of bridges using GPS/Accelerometers, PhD Thesis, Institute of Engineering Surveying and Space Geodesy, the Nottingham University, UK, 239 p., May 2002.

[7] M.R. Kaloop, H. Li, Monitoring of bridges deformation using GPS technique, KSCE J. Civ. Eng. (KSCE) 13(6), pp. 423-431, 2009.

[8] M.R. Kaloop, D. Kim, GPS-structural health monitoring of a long span bridge using neural network adaptive filter, Survey Review, Vol. 46, No. 334, pp. 7-14, 2014

[9] M. Yu., H. Guo, C. Zou, "Application of wavelet analysis to GPS deformation monitoring." Proc. of IEEE/ION PLANS, San Diego, California, pp. 670-676, 2006. 